\title{
Dermic diffusion and stratum corneum: A state of the art review of mathematical models
}

\author{
Ana Couto, Rúben Fernandes, M. Natália S. Cordeiro, Sara S. Reis, \\ Rogério T. Ribeiro, Ana M. Pessoa
}

\begin{abstract}

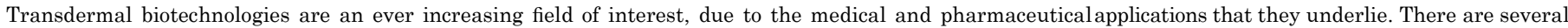

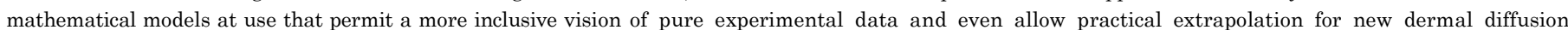

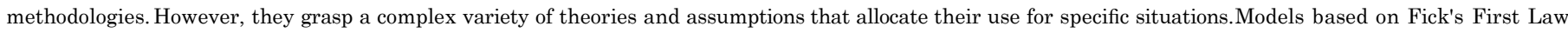

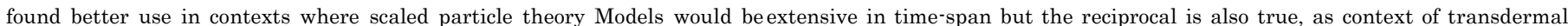

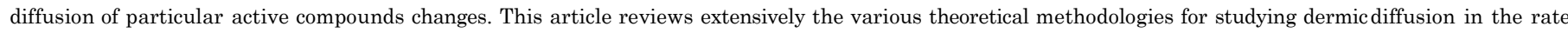

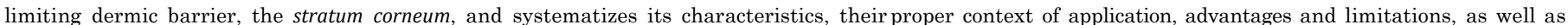
future perspectives.
\end{abstract}

\section{Keywords:}

Transdermal diffusion, Mathematicaldiffusion models, Fick's Law, Sensors, Hybrid approach

\section{Introduction}

Nanotechonology, whose importance is presently undisputed [1,2] represents the avenue and the frontier of Biotechnology and Biomedical Engineering. Among many of the possible new solutions, the ones 
regarding disadvantages of current biomedical devices that increase transport across the skin, such as microneedles [2,3], are of particular interest in transdermal applications. At present, microneedles, a device that may provide a more efficient transdermal drug delivery, are an example of a larger research area: dermic diffusion. The microneedle piercing increases the skin permeability of the variety of compounds ranging from low molecular weight tracers to proteins, DNA and even nanoparticles $[4,5]$. However, the trauma suffered by the patients is still an issue, and a nanoneedle could prevent such problems, since there are studies relating positively the narrowness of the needle size and the minimization of trauma [6]. The common option of microneedles in many transdermal applications, despite the abovementioned issues, lies in the difficulty to diffuse the compounds through the skin, especially through the stratum corneum $[7,8]$. Understanding this specific diffusion means understanding the ground that sustains drug delivery method's evolution. However, it is difficult, if not impossible in some cases, to determine experimentally the nanoidiosyncrasies at molecular level that explain the sustainability of the material and/or device at macroscopic level. Furthermore, the apprehension at molecular level of the material's nature and chemical reactivity would permit extrapolation for other scenarios and even new materials. The importance of theoretical models, thus, is increasingly paramount not only for the present micro technologies but also for the future achievements in the nanoresearch.

Nevertheless, the elaboration of such models faces the complexity of the dermic barrier. To study such surface implies a large number of individual fragments, each one with a unique nano chemical composition. The skin layer that presents the first barrier in dermic diffusion is the stratum corneum (SC), just 15 to $20 \mu \mathrm{m}$ thick, and is constituted by differentiated keratinocytes embedded in lipid layers [3]. Not only is the SC complex in merely chemical constitution but also its organization, an inherent aspect of the identity of the SC [7], adds to the difficulty of modelling the barrier. Diffusion models usually focus on the SC since, though it is the thinnest layer at most locations of diffusion interest, it presents the highest resistance to transport and is, therefore, rate limiting for most solutes $[8,9]$. Beneath the SC is the location of the epidermis, which thickness ranges from 130 to $180 \mu \mathrm{m}$ and finally, as one penetrates the skin, one founds the dermis, filled with complex structures such as the nerves, blood vessels, nociceptors, lymph vessels, hair follicles and sweat glands [10,11], as schematized in Fig. 1 .The dense multilayered nature of dermic barrier, thus, complicates the (trans) dermal drug delivery, even when the administration is by simply piercing through the skin. This explains why the majority of (trans) dermal products in the market are low molecular-weight drugs with high potency [12-14].

This article aims to comprehensively review the diffusion mathematical models through the SC. Though active particles may be delivered through other processes, diffusion is considered to be the essential process of drug transport via the SC, if not even the primary

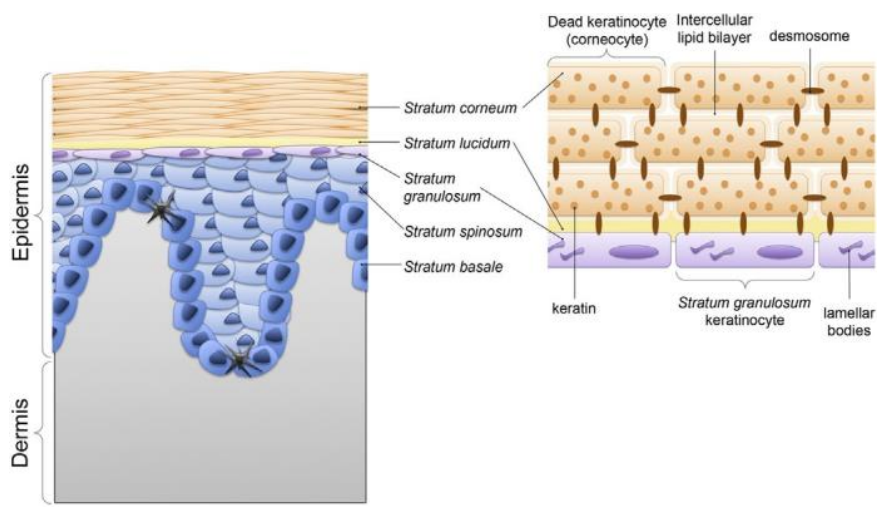

Fig. 1. Stratum corneum.
$[8,9]$. Thus, we will not consider any other transdermic drug transport, which has been reviewed elsewhere $[15,16]$. The following processes exemplify the potential applications of transdermal diffusion mathematical models, in view of the many experimental limitations at present.

\section{Transdermal applications}

Transdermal application is a route of administration wherein active compounds are delivered across the skin for systemic distribution. $\mathrm{Al}^{-}$ though the skin is a large and logical target for drug delivery, its basic functions, as a protective barrier against external pervasive agents, limit its utility. Therefore, understanding the barrier's functions and namely its flexibility, provides the basis of applications, as described below.

\subsection{Diffusion of active compounds}

Drug delivery through the skin has multiple benefits over both oral route and conventional injections, although the dense structure of the stratum corneum and the limited number of hair follicles and sebaceous glands complicates the delivery of therapeutic doses [2].

The dermis and the overlying epidermis compose the skin. The outermost layer of the epidermis is the stratum corneum, comprised of a multi-layered structures of keratinocytes embedded in a matrix of hydrophobic lipids organized into tight lamellar structures [17,18]. The dermis contains a network of capillaries that connect to the systemic circulation and if a drug penetrates the stratum corneum, then it can readily reach the dermis and enter the bloodstream by passive diffusion. Diffusion is considered the rate-limiting step for the transdermal transport of drug molecules, through the delivery of molecules into and across the skin and depends on the substance's physicochemical properties.

The particles, which are described to have a better drug delivery, are the nanoparticles. They enhance drug absorption allowing sustained drug release for a prolonged period of time and protecting the capsulated substance from chemical degradation. The active compounds can enter into the skin by different routes: intercellular pathway, transcellular pathway, transappendageal pathway and paradermic pathway.

Whereas the active compounds enter the skin through the lipid extracellular matrix (ECM) in the (1) intercellular pathway, occupying the intercellular spaces of the keratinocytes, in the (2) transcellular pathway which is through the keratinocytes; finally, the active compounds may also cross along the shafts of hair follicles in the (3) transappendageal pathway, through the sebaceous and sweat glands that are dispersed all over the skin. Nanocarriers can enter into the skin and remain intact or can be degraded near the skin surface releasing the active substance to penetrate into the skin layers $[18,19]$.

\subsection{Removal of active compounds/substances}

Drug delivery involves a number of circumstances including the possibility of extracting the active compounds; one example is the extraction of glucose and lactate [20,21]. Using transdermal reverse iontophoresis allows the simultaneous extraction of glucose and lactate from human subjects.

Transdermal monitoring using iontophoresis is made possible by the skin's permeability to small molecules and the nanoporous and microporous nature of the structure of the skin. Reverse iontophoresis refers to the passage of a low level of current through the skin, normally via a pair of skin electrodes containing a conducting gel, to promote the extraction of both charged and neutral molecules [20].

Ching and collaborators using a nanoporous membrane as the skinlike barrier in the iontophoresis cell with and without application of iontophoretic current performed in vitro diffusion studies. This showed that passage of a current facilitates movement of lactate and glucose across 
the nanoporous membrane when compared to ordinary diffusion; and during the studies, lactate and glucose were extracted successfully using reverse iontophoresis [21].

\subsection{Dermal reconstruction/skin regeneration}

Dermal reconstruction is a complex process, which involves many cells, such as epidermal, fibroblastic, and endothelial cells, and various processes such as proliferation, cell migration, matrix synthesis, and contraction. Wound healing is a complex and dynamic process of restoring cellular structures and tissue layers which progresses in an arranged and conventional fashion such as inflammation (reactive), proliferation (regenerative) and maturation (remodelling) [22].

When the skin is breached, initially there is the laying down of a fibrin blood clot, which plugs the defect and seals off the underlying tissues from oxygen in the air. The degenerating platelet secretes platelet-derived growth factor (PDGF) and stimulates the first phase of cell proliferation of epidermal cells, fibroblasts and endothelial cells. Then, neutrophils arrive at the wound site and clear out debris and contaminating bacteria and secrete proinflammatory cytokines, which activate local fibroblasts and keratinocytes. Past few days, the blood-borne macrophages replace the neutrophils and they produce cytokines and growth factors essential to wound healing. After a week, the wound is infiltrated by activated fibroblasts, stimulated by TGF- $\beta$ that increases the synthesis of single strands of collagen. Then wound fibroblasts are transformed into myofibroblasts, which express $\alpha$-smooth muscle actin ( $\alpha$-SMA) acquiring the ability to generate strong contractile forces. The proliferation of myofibroblasts results in the formation of scar tissue [23].

\section{Dermicdiffusionandmathematicalmodels: a review}

Traditionally, the skin, the SC and the diffusion phenomena have been studied associated with pure mathematical models, especially based on the Fick Equation [24-32]. A systematic analysis of these models implies a division based upon the particular approach and use of the Fick Law. However particular views in specific devices have raised new challenges and other mathematical models were developed. An extensive review of these models was made and the relevant synthesis is presented in Table 1.

The mathematical models that approach the skin at nano level can be classified according to the methodological assumptions, i.e., they are either basedon the Fick's FirstLaw, the Laplace transform, the scaled particle theory, the homogeneous membrane model or hybrid approach (using the First and the Second Law of Fick). Thus, analysing each model implies an analysis of the particular approach chosen and the reasons for such choice. Also, it is important to understand the boundary condi-

tions in these models. Boundary conditions are the physical restraints in the mathematical model, limiting the solution-space of the equations.

\subsection{Fick's First Law}

When a drug is placed on the skin surface, it migrates to the innermost layers through the stratum corneum and viable epidermis [29]. Stratum corneum, as depicted in Fig. 1, is composed by keratinocytes which becomes a substantial barrier to drug delivery because active and facilitated transport is difficult in delivering drug [3]. Passive diffusion is a mechanism by which chemicals move through the stratum corneum, as can be seen in Fig. 3 [32].

Fick's Laws of diffusion determines that diffusion occurs in favour of the concentration gradient, in others words, it occurs in the direction from higher to lower concentration [32,33]. This principle is applied in recent mathematical models used to describe the dermal absorption through the stratum corneum $[32,34,35]$. The stratum corneum lipid matrix is viewed as a homogeneous medium surrounding keratinocytes where a substance diffuses from high to low concentration according to
Fick's First Law. The fraction that is absorbed in specific period of time is constant and will remain constant at different concentrations [32,34].

Let us consider that $Q$ is the amount of solute, i.e., the amount of substance that will flow in a small interval of time through a small surface. If we consider the small surface, $A$, as the skin membrane where the diffusion occurs, over a period of time $t$, through the path length $h$ (the thickness of the skin membrane), due to the concentration gradient across the two interior surfaces of the skin, $\nabla C s$ then the relation between $Q$ and the diffusion's coefficient in the skin membrane, $D$, is of direct proportional and is given by Fick's First Law, describing skin transport under steady-state conditions [38]:

$$
Q=D \frac{A}{h} \mathrm{t} \nabla C_{s} .
$$

This approach assumes that dermal absorption is a first order process that can be described according to Fick's First Law of diffusion [32]. Stratum corneum behaves like a pseudo-homogenous membrane and barrier properties do not vary with time or position. Defining steady-state skin flux, Jss as the amount of solute per unit area and per unit time, Eq. (1) is rearranged as follows:

$$
\mathrm{kss}=\frac{Q}{A t}=\frac{D \nabla C_{s}}{h}
$$

Whereby, the maximum flux, Jmax, is given when the maximum solubility $S$ of a solute in the stratum corneum is achieved, and the equation can be written as $[30,36]$.

$$
\max =\frac{D S_{s}}{h} .
$$

The thermodynamic activity for any given solute is defined by the fractional solubility of the solute in the skin $\left(C_{s} / S_{s}\right)$. However, nonlinearity can arise as a result of solute-skin and solute-vehicle non-ideal interactions [30,37]. It is $\left(C_{v}\right)$ more convenient to express concentrations in terms of the solute concentration in the vehicle and a partition coefficient, $K$, of the solute between the skin and the vehicle as follows:

$$
\begin{aligned}
& \text { fss }=\frac{K D \nabla C_{V}}{h} \\
& \text { where } \\
& K=c_{i} / C_{v}
\end{aligned}
$$

The assessment of permeability coefficient $\left(\mathrm{K}_{\mathrm{p}}\right)$, has been the main focus of the permeation model. $K_{p}$, is defined as the steady-state flux of chemical across the skin $\left(J_{s s}\right)$ normalized by the concentration gradient, $C_{v}$ :

$$
\mathrm{K}_{\mathrm{p}}=\frac{J s s}{\nabla C_{v}} .
$$

The understanding of percutaneous penetration is best exemplified in models that are underpinned by experiment. For example, the concentration profiles in the skin have been examined to separate the effects of the partition coefficient and the diffusion on permeation [38]. Furthermore, mathematical models provide means to assess the likelihood of systemic exposure upon dermal contact with toxic chemicals. Mitragotri and collaborators have been applied of mathematical modelling to dermal risk assessment, privileging the use of permeability coefficients $\left(\mathrm{K}_{\mathrm{p}}\right)$ and their model-based estimation [30]. 
Table 1

Mxhematical modeds for dermic diffusion.

\begin{tabular}{|c|c|c|c|}
\hline Moded & Theore bical approadh & Mathematical model & Validation \\
\hline Fod's First Law & $\begin{array}{l}\text { Sratum corneum lipid matr ix is viewed as } \\
\text { a homo geneous medium surrounding } \\
\text { carneocyles which the chemical proclud } \\
\text { diffises from high to low conoentration } \\
\text { xording to Ficks First law. }\end{array}$ & 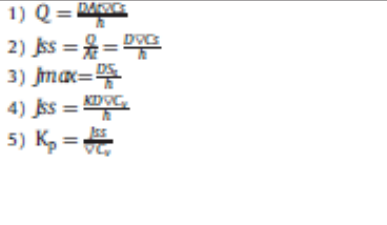 & 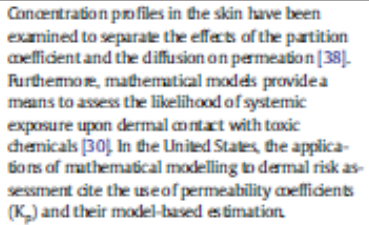 \\
\hline $\begin{array}{l}\text { Fodss First Law passive } \\
\text { diffusion model }\end{array}$ & $\begin{array}{l}\text { Models of passive diffusion are based on } \\
\text { cinservation of the number of the partides } \\
\text { and Ficks Laws of diffusion. }\end{array}$ & 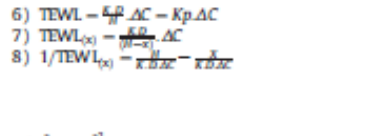 & 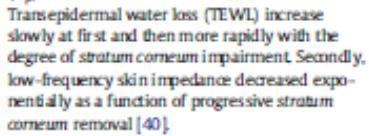 \\
\hline Fids Second LaW & $\begin{array}{l}\text { The system is modelled } \approx \text { a one- } \\
\text { dimensional transient diffusion system by } \\
\text { using a chemical penetr sion enhance- } \\
\text { ment (oleicacid, OA). }\end{array}$ & 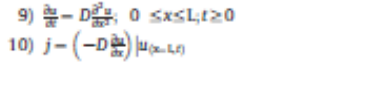 & $\begin{array}{l}\text { In the presence of the penetration enhancer, the } \\
\text { diffusion and permeation of model drugs were } \\
\text { mare rapidly than for the stratum comeum lipid } \\
\text { mode menbrane without } \mathrm{OA}[25]\end{array}$ \\
\hline $\begin{array}{l}\text { Fodrs Secand Law passive } \\
\text { diffusion model }\end{array}$ & $\begin{array}{l}\text { The systems are represented by a velicle, a } \\
\text { skin membrane and a capil lary. }\end{array}$ & 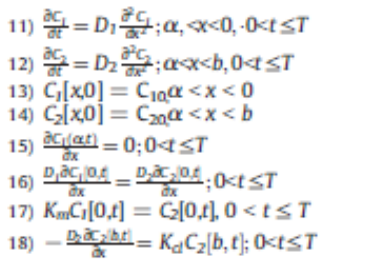 & $\begin{array}{l}\text { This tedınique can be applied to es timate the } \\
\text { dase--lading neossary to adtieve a specified re- } \\
\text { lease rate. A canstant plasma drug concentraion } \\
\text { is obtained increasing the application period and/ } \\
\text { or reducing the removal per iod which produce a } \\
\text { mare continuous drug delivery [28] }\end{array}$ \\
\hline Hybrid approxh & $\begin{array}{l}\text { As previously mentioned, current } \\
\text { mxihemxical modes use the Fidks law of } \\
\text { diffision b describe dermal atsorption } \\
\text { throught the stratum conneum. }\end{array}$ & 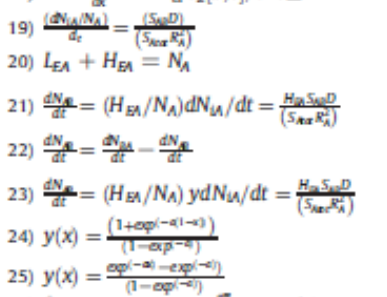 & 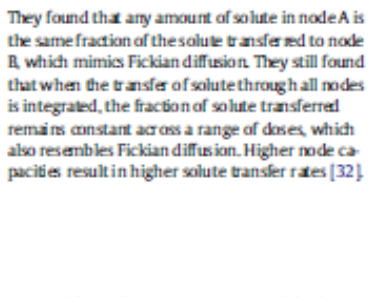 \\
\hline Laplace trans form & $\begin{array}{l}\text { Laplace transform can be wed to describe } \\
\text { skin permeatian of drug and metabolite. } \\
\text { slmitting first arder metabolism kinetics }\end{array}$ & 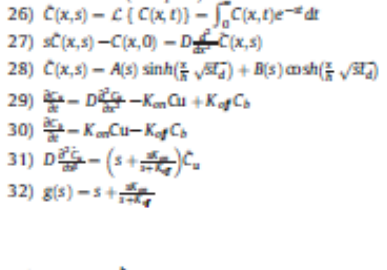 & 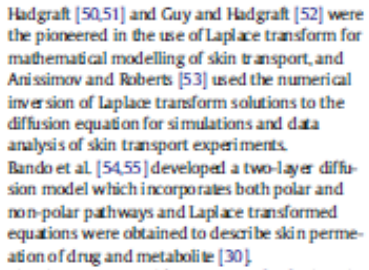 \\
\hline Scaled partide theory & $\begin{array}{l}\text { The partibon coefficient between the } \\
\text { stratum comeum and the vehide is } \\
\text { estimied fram structure-activity } \\
\text { igarithms relat ing experimental } \\
\text { measurement. }\end{array}$ & 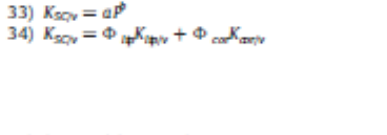 & 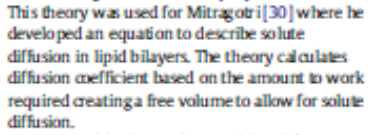 \\
\hline $\begin{array}{l}\text { Homogeneous membrane } \\
\text { model }\end{array}$ & $\begin{array}{l}\text { Study of the transient diffusion through } \\
\text { model stratum carneum structures } \\
\text { including both arderet and disordered } \\
\text { keratinocyles phases. }\end{array}$ & 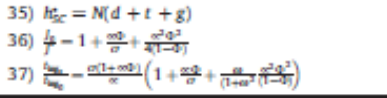 & $\begin{array}{l}\text { Eq (37) and (39) were been validxed for } \\
\text { isotropic lipids and impermeable keratinocytes } \\
\text { [68. }\end{array}$ \\
\hline
\end{tabular}

\subsubsection{Fick's First Law and passive diffusion models}

Models of passive diffusion are based on particle number conservation and diffusion Fick's Laws. Some reported mathematical approaches reported are based on diffusion models that consist in partial differential equations describing drug delivery in space and time according with diffusion Fick's Laws [31]. According to diffusion Fick's Laws, penetrants are transported, through the skin, via the transcellular, intercellular and transappendageal routes $[18,28,39]$. These different transports are depicted in Fig. 2. 


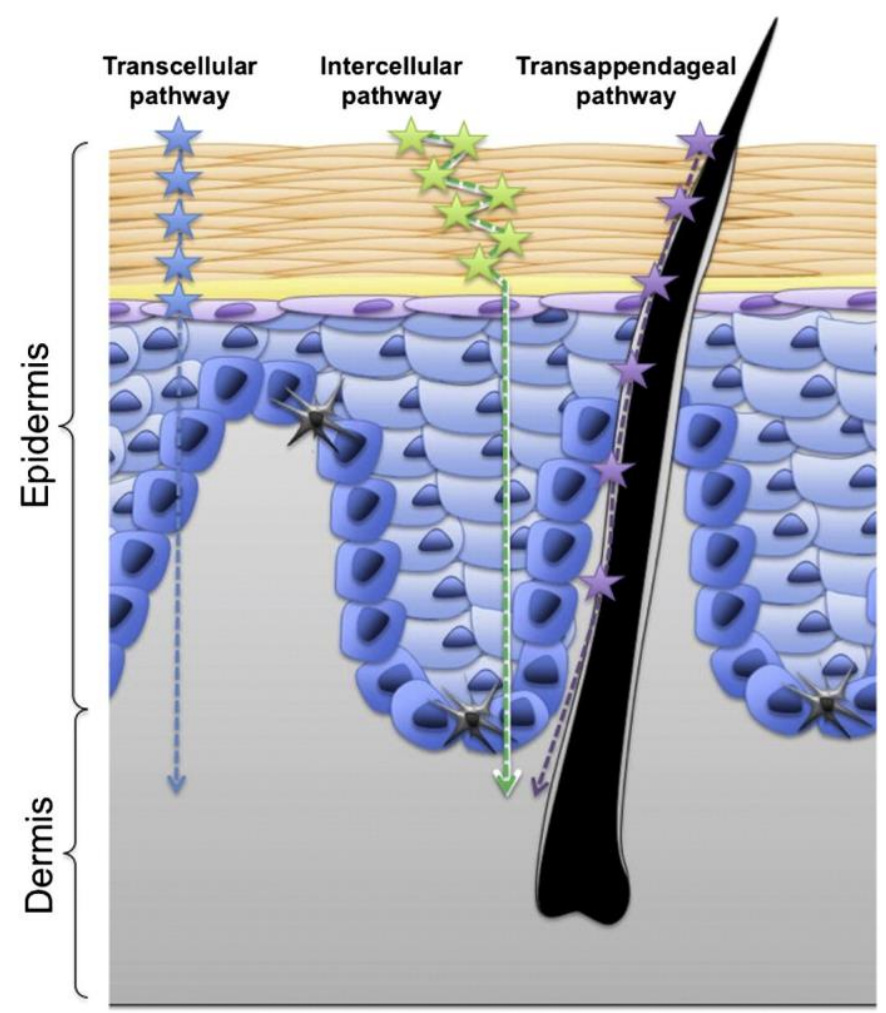

Fig. 2. Schematic presentation of the routes for permeation of the agent applied.

Regarding the studies of Mitragotri and collaborators, the transport processes in the skin can be described by Fick's First Law [30]. Furthermore, Higuchi and team derived mathematical models that describe percutaneous absorption as a passive diffusion process in the vehicle and the membrane layers in series [36].

Sekkat and collaborators evaluated the biophysical properties, such as transepidermal water loss (TEWL) of porcine ear skin and compared the results with data from the human skin in vivo [40]. Using Fick's First Law of diffusion to describe TEWL across the skin, if $K$ is the stratum corneum/water partition coefficient, $D$ is the diffusivity of water across the stratum corneum of thickness $H$, and $\Delta C$ is the water concentration

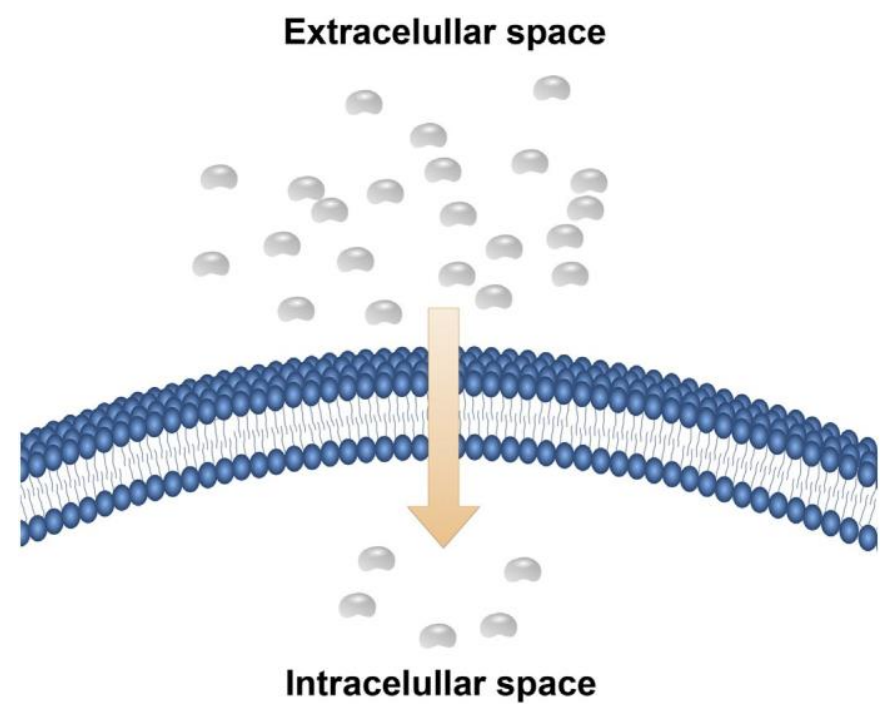

difference across the barrier (in $\mathrm{g} / \mathrm{mL}$ units), then TEWL is directly proportionate to the water concentration difference across the barrier:

$$
\mathrm{TEWL}=\frac{K D}{H} \cdot \Delta C=\mathrm{K}_{\mathrm{p}} \Delta C
$$

The combined parameter $\mathrm{K}_{\mathrm{P}}=(K . D) / H$, is the permeability coefficient. Determination of $H, D$ and $\mathrm{K}_{\mathrm{P}}$ comes from: (1) knowledge or assumption of $K$, and (2) construction of a plot of $1 / \mathrm{TEWL}_{(\mathrm{x})}$ versus $X$, where $X$ (in $\mu \mathrm{m}$ units) is part of the stratum corneum removed by tape stripping, resulting in TEWL increasing to $\operatorname{TEWL}_{(\mathrm{x})}[40,41]$. The equations used in the plot analysis are:

$$
\begin{aligned}
& \operatorname{TEWL}_{(x)}=\frac{K . D}{H-x} \cdot \Delta C \\
& 1 / \operatorname{TEWL}_{(x)}=\frac{H}{K \cdot D \cdot \Delta C}-\frac{X}{K \cdot D \cdot \Delta C} .
\end{aligned}
$$

TEWL increased slowly at first and then more rapidly with the degree of stratum corneum impairment. Secondly, low-frequency skin impedance decreased exponentially as a function of progressive stratum corneum removal. The differential sensitivities of TEWL and lowfrequency impedance measurements to the degree of barrier impairment suggest that the techniques used together represent a way to characterize stratum corneum functionality [40].

\subsection{Fick's Second Law}

Other mathematical models describing the delivery of drug through the dermis are based on Fick's Second Law. While Fick's First Law assumes steady state, Fick's Second Law predicts the concentration gradient changes, through diffusion, with time. Any dermic diffused drug is placed on the skin surface and travels to the innermost layer through the stratum corneum and viable epidermis, as previously seen. One can locate the origin of the delivery process at the interface between these two regions [29].

According Fick's Second Law of diffusion, to estimate the concentration of the drug along a perpendicular axis to the membrane, designed normally by $u(t, x),[24,25,29]$, where $x$ is the thickness of the membrane and $t$ is the period of time, it must be found the solution of following linear one-dimensional diffusion equation:

$$
\frac{\partial u}{\partial t}=D \frac{\partial^{2} u}{\partial x^{2}} ; 0 \leq x \leq L ; t \geq 0
$$

where the thickness varies from null to a maximum, L, corresponding to the maximum value of the membrane thickness. The delivery rate or flux, $j$, is defined by:

$$
j=\left(-D \frac{\partial u}{\partial x}\right) \mid u_{(x-1, t)}
$$

In addition to physical methods, drug permeation through the stratum corneum can use chemical penetration enhancers [25,42,43]. Cis-9-Octadecenoic acid (oleic acid, OA) was found to be a good penetration enhancer $[25,44,45]$, and research regarding the impact of the penetration enhancer, oleic acid, on the barrier properties of stratum corneum lipid model membranes was conducted.

The Second Law was studied using a two lipid model membranes (membrane I and membrane II) with incorporated oleic acid. The composition of the membranes is summarized in Table 2 , and was characterized the impact of their composition on the barrier function by conducting diffusion and permeation studies of three model molecules (urea, caffeine and diclofenac sodium).

The study showed that the presence of oleic acid weakened the barrier function of membrane I and demonstrated the opposite effect 
Table 2

Composition of SC lipid model membranes.

\begin{tabular}{ll}
\hline Model membrane & Composition \\
\hline Membrane I & Cer $[\mathrm{AP}] / \mathrm{Chol} / \mathrm{PA} / \mathrm{ChS}$ \\
Membrane II & $\mathrm{Cer}[\mathrm{AP}] / \mathrm{Cer}[\mathrm{EOS}] / \mathrm{Chol} / \mathrm{PA}$ \\
Membrane Ia & $\mathrm{Cer}[\mathrm{AP}] / \mathrm{Chol} / \mathrm{PA} / \mathrm{ChS} / \mathrm{OA}$ \\
Membrane IIa & $\mathrm{Cer}[\mathrm{AP}] / \mathrm{Cer}[\mathrm{EOS}] / \mathrm{Chol} / \mathrm{PA} / \mathrm{OA}$ \\
& \\
& \\
\hline
\end{tabular}

on membrane II. In the presence of the penetration enhancer the diffusion and permeation of model molecules were more rapid than for the stratum corneum Lipid Model Membrane without oleic acid.

Previous studies showed that: (1) the diffusion of urea was faster than the permeation of caffeine and diclofenac sodium; (2) urea, the most hydrophilic drug, diffused faster than caffeine, the more lipophilic molecule; and (3) urea is even faster than the most lipophilic drug, diclofenac sodium [25].

Several authors used a model based on Fick's Second Law of diffusion to calculate the diffusion coefficient of model drugs. Thus, the drug concentration, $u(t, x)$, within the model membrane can be approximated by a solution of linear one-dimensional diffusion equation, as mentioned before [24,25].

\subsubsection{Fick's Second Law and passive diffusion models}

The Fickian diffusion model is also applied in studies regarding the use of transdermal patch with multi-layer, planar, drug-in-polymer adhesive patch applied to the skin, where each layer contains a different concentration of drug encapsulated in the polymer matrix. It can also be seen in cases where the systems are represented by a vehicle, a skin membrane and a capillary [28]. In this model, drug concentrations in the vehicle, $C_{1}$, and skin, $C_{2}$, are different, as are the effective drug diffusions on the vehicle and the skin, respectively; $D_{1}$ and $D_{2}$

$$
\begin{aligned}
\frac{\partial C_{2}}{\partial t} & =D_{2} \frac{\partial^{2} C_{2}}{\partial x^{2}} ; a<x<b, 0<t \leq T \\
\underline{\partial C}_{1} & \frac{\partial C_{1}}{\partial t}=D_{1} \frac{\partial^{2} C_{1}}{\partial x^{2}} ; a<\ll<0,0<t \leq T .
\end{aligned}
$$

The thicknesses of the vehicle, $a$, and of the skin, $b$, are part of the initial conditions of Eqs. (11) and (12). Let $\mathrm{C}_{10}$ be the initial drug concentration in the vehicle and $\mathrm{C}_{20}$ be the initial drug concentration in the skin for the first application, both in $\mu \mathrm{g} / \mathrm{cm}^{3}$. Then the initial conditions are:

$$
\begin{aligned}
& C_{1}(x, 0)=C_{10}, a<x<0 \\
& \text { and } \\
& C_{2}(x, 0)=C_{20}, a<x<b .
\end{aligned}
$$

The boundary conditions are represented by Eqs. (15), (16), (17) and (18). Considering that $K_{m}$ is the patch-skin partition coefficient, $K_{c l}$ is defined as the clearance per unit area of the medicament per

unit concentration excess at $x=b, T$ is the application period, and that the transport is done in the direction perpendicular to the skin surface, the Eq. (15) states that no exchange of material occurs between the vehicle and the surrounding, while Eq. (16) imposes the continuity of flux across the vehicle/skin interface, Eq. (17) corresponds to an equilibrium condition at the vehicle/skin interface and Eq. (18) states that elimination of drug by capillary system follows first-order kinetics at $x=b .[28,46]$

$$
\begin{aligned}
& \frac{\partial C_{1}(a, t)}{\partial x}=0 ; 0 \varangle \leq T \\
& \frac{D_{1} \partial C_{1}(0, t)}{\partial x}=\frac{D_{2} \partial C_{2}(0, t)}{\partial x} ; 0 \triangleleft t \leq T
\end{aligned}
$$

$$
K_{m} C_{1}(0, t)=C_{2}(0, t), 0 \triangleleft t \leq T
$$

$$
-\frac{D_{2} \partial C_{2}(b, t)}{\partial x}=K_{d} C_{2}(b, t) ; 0<\leq T .
$$

Simon and team have dedicated efforts to study the transport of a medicinal agent through the vehicle and subsequent penetration into the stratum corneum [28]. Eigenmodes and eigenvalues were computed and introduced into an inversion formula to estimate the delivery rate and the amount of drug in the vehicle and the skin. The calculations were obtained using Mathematica ${ }^{\circledR}$ (Wolfram Research, Inc.), a computer software.

It was observed that repeated applications of a fixed dose lead to an accumulation of drug in the skin. This accumulation is counterbalanced by an increase in the flux of the drug to the receptor site $[28,46]$. A plateau is reached when the rates of absorption and elimination are equal. This technique can be applied to estimate the dose-loading necessary to achieve a specified release rate. A constant plasma drug concentration is obtained by increasing the application period and/or reducing the removal period which produce a more continuous drug delivery [28].

\subsection{Hybrid approach: first and second Law of Fick}

As previously mentioned, current mathematical models use the Fick's Law of diffusion to describe dermal absorption through the stratum corneum $[32,34,35]$. Chemicals, in the stratum corneum lipid matrix, diffuse from high to low concentration according to Fick's First Law. The fraction of a chemical dose that is absorbed through the skin over a specified period of time is constant, and will remain constant at various concentrations of chemical applied in the skin surface.

Schumm and collaborators [32] propose another approach to the

mathematical modelling of the chemical kinetics in the stratum corneum lipid matrix. They represented the lipid structure as a network where the lipid regions are represented as nodes and used the following models to describe the process by which solute transfers between two adjacent nodes, based on Fick's Law.

The first model is designed to have a linear solute transfer. To node A with $N_{A}$ molecules, a fraction of its molecule will be at the A-B interface during each time interval as a function of the relative interface sizes and the diffusivity of the solute. Consider that $N_{A}$ is the molecules in node A, $d N_{i A}$ is the number of solute molecules within node A migrating to the interface of nodes $\mathrm{A}$ and $\mathrm{B}$, during the time interval $d_{t} S_{A B}$ is the surface area shared by nodes A and B, $S_{A t o t}$ is the total surface area of node A, $D$ is the solute diffusivity in node $\mathrm{A}$, and $R_{A}^{2}$ is the radius of the region represented by node $\mathrm{A}$. Then the fraction of molecules at the A-B interface during each time interval is a function of the relative interface sizes and the diffusity of the solute:

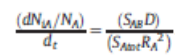

(19)

The molecules in node A at the interface of node A with $\mathrm{B}$ will partition to node B if they have sufficient energy to cross the node boundary. The Maxwell-Boltzmann distribution (exemplified in Fig. 4) characterizes the energy of a group of molecules. The molecules with higher energies will be on the right side of the distributions and the lower energy will be on theleft.

The total area under the distribution is the number of solute molecules in node $\mathrm{A}$.

$$
L_{E A}+H_{E A}=N_{A} .
$$




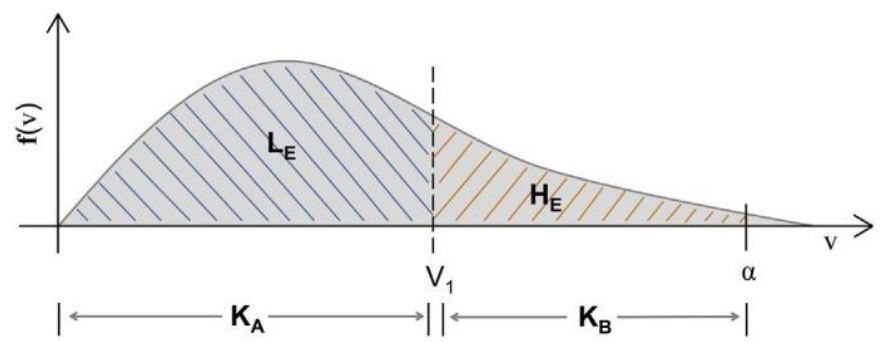

Fig. 4. Maxwell-Boltzmann distribution: $L_{E}$, Low energy molecules; $H_{E}$, High energy molecules; $K_{A(B)}$, solubility of node A (B); $\alpha$, upper limit of integration for $N_{A} ; V_{1}$, upper limit integration for $L_{E}$.

The molecules that partition from node A to node B are the molecules at the A-B interface sufficient energy to cross the node boundary.

$$
\frac{d N_{A B}}{d t}=\left(H_{E A} / N_{A}\right) d N_{M S} / d t=\frac{H_{E A} S_{A B} D}{\left(S_{A \alpha X} R_{A}^{2}\right)} .
$$

The solute level of node $\mathrm{A}$ is then update with the net difference of the transfer between nodes A and B.

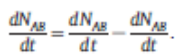

(22)

The second model is designed to increase solute transfer from nodes with a high to low solute concentration to low concentrations. Higher solute concentrations cause physical-chemical changes in the lipid matrix that decrease its barrier properties. When the quantify $\mathrm{A}$ is greater than B, diffusivity in A will be greater than diffusivity in B. The difference induces a gradient increasing the transfer from node A to node B. And the third model is designed to enforce a node capacity and allow only a finite amount of solute in a node at any given time [32]. This model calculates the fraction $y$ to transfer from node A to node B with the fraction $x$ of $N_{B}$ over $N_{B \max }$

$$
\frac{d N_{A B}}{d t}=\left(H_{E A} / N_{A}\right) y d N_{h} / d t=\frac{H_{E A} S_{M B} D}{\left(S_{A x} R_{A}^{2}\right)} .
$$

The equation $y(x)$ is given for a rolloff model and an exponential model. In the rolloff the transfer as node $B$ reaches its capacity (when $x$ tends to 1 ) and in exponential model exponentially decays the transfer as node $\mathrm{B}$ reaches its capacity.

$$
\begin{aligned}
& y(x)=\frac{\left(1+\exp ^{(-a(1-x))}\right)}{\left(1-\exp ^{-a}\right)} \quad \text { (rolloff mode) } \\
& \text { and } \\
& y(x)=\frac{\exp ^{(-a x)}-\exp ^{(-a)}}{\left(1-\exp ^{(-a)}\right)}
\end{aligned}
$$

Schum and team simulated the basic models over time and differing surface solute concentrations and they found that any amount of solute in node $\mathrm{A}$ is the same fraction of the solute transferred to node $\mathrm{B}$, which mimics Fickian diffusion. They also found that when the transference of solute through all nodes is integrated, the fraction of solute transferred remains constant across a range of doses, which also resembles Fickian diffusion. Higher node capacities result in higher solute transfer rates [32].

Furthermore, Siepmann and collaborators mentioned several theories based on diffusion Fick's Law, and conclude that the mathematical treatment can be rather straightforward, if drug release is controlled with constant diffusion coefficients [26]. They distinguished two types of systems: reservoir system and monolithic systems, as illustrated by Fig. 5. The reservoir system is surrounded by a release rate controlling barrier membrane. In the monolithic system there is no local separation between a drug reservoir and the release rate controlling barrier [26].

In the reservoir device, if initial drug concentration is below drug solubility, released drug molecules are not replaced and the drug concentration at the inner membrane's surface decrease with time. Still, if the initial drug concentration is higher than the drug solubility, release molecules are replaced by the (partial) dissolution of drug crystal/ amorphous aggregates, resulting in constant drug concentrations at the inner membrane's surface. In the monolithic device, the system geometry has influence on the resulting drug release kinetics. If the initial concentration is below drug solubility, the drug molecules are dissolved within the carrier material. If not, dissolved drug molecules coexist with amorphous aggregates and/or drug crystals [26,47].

\subsection{Laplace transform}

The expression of the transport of a solute through the skin involves a number of steps in a space and time variant process that it is not easy. It is not straightforward to describe the process in a single equation due

to the complexity of the equations [30]. Laplace transform can be used to describe skin permeation of drug and metabolite, admitting first orderkinetics.

Laplace transform is an integral transformation used to resolve ordinary and partial differential equations [25,30,31,48,49]. Laplace transform is defined by:

$$
\hat{C}(x, s)=\mathcal{L}\{\mathcal{C}(x, t)\}=\int_{0}^{\infty} C(x, t) e^{-s t} d t
$$

where Lis the Laplace operator, $s$ is the Laplace variable and the hat over the function denotes the Laplace transform.

Laplace transform reduces the partial differential diffusion equation into an ordinary differential equation which is much easier to solve, and his way, the Laplace transform of the diffusion equation, where $C(x, 0)$ is the initial condition, is asfollowing:

$$
s \hat{C}(x, s)-C(x, 0)=D \frac{d^{2}}{d x^{2}} \hat{C}(x, s) .
$$

If the skin starts out without any chemical, $C(x, 0)=0$. Taken that into account and considering $h$ to be the thickness of the SC, $t_{d}=h^{2} / D$ is the characteristic time of diffusion, and $A(s)$ and $B(s)$ functions of

\section{Reservoir system}
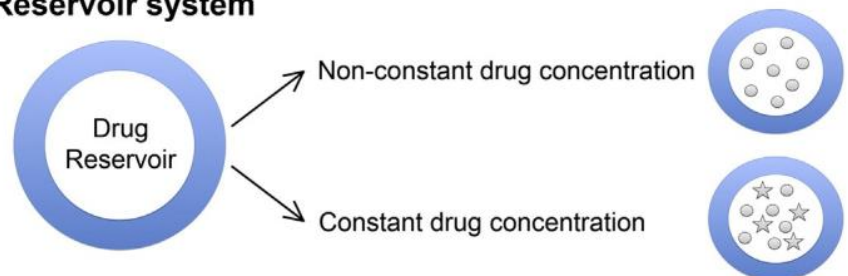

\section{Monolithic system}

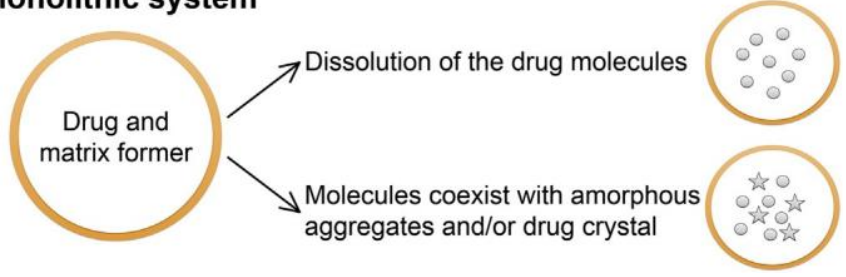

Fig. 5. Representation of reservoir and monolithic system. 
only $s$, only determined from boundary conditions, the above equation presents the following general solution:

$$
\hat{C}(x, s)=A(s) \sinh \left(\frac{x}{h} \sqrt{s t_{d}}\right)+B(s) \cosh \left(\frac{X}{h} \sqrt{s t_{d}}\right)
$$

Also, $h$ can be further manipulated to calculate the transdermal flux and cumulative drug transport [30,50-52].

Simon and collaborators indicated that the equations of drug delivery can be solved using the Laplace transform [29]. The Laplace transformbased solution strategy was chosen due to the direct calculation of an effective time constant. This time constant is the time it takes to achieve a desired release rate and steady-state concentrations in the skin.

Roberts and team also considered the kinetics associated with the reservoir effect of the stratum corneum [37]. As the fraction unbound is less than unity, the link leads to a slower diffusion. If binding/partitioning is not fast compared to diffusion, the single diffusion equation has to be replaced by coupled partial differential equations:

$$
\begin{aligned}
& \frac{\partial C_{u}}{\partial t}=D \frac{\partial^{2} C_{u}}{\partial x^{2}}-K_{a n} C u+K_{o f f} C_{b} \\
& \frac{\partial C_{b}}{\partial t}=K_{a n} C u-K_{a y f} C_{b}
\end{aligned}
$$

where $C_{b}$ and $C_{u}$ are the concentrations of bound and unbound solutes, $D$ is the diffusion coefficient if the unbound solute (bound solute is assumedimmobile, or its diffusion is so slow that it can be neglected) and $K_{o n}$ and $K_{\text {off }}$ are the binding and unbinding rate constants.

Taking Laplace transform of these equations and expression of $\ddot{C}_{b}$ using $\ddot{C}_{u}$ yields:

$D$

$$
D \frac{\partial^{2} \hat{C}_{u}}{\partial x^{2}}=\left(s+\frac{s K_{o n}}{s+K_{\text {aff }}}\right) \hat{C}_{u}
$$

The only difference between simple diffusion equation in the Laplace domain is that $s$ is replaced by a function $g(s)$, where:

$$
g(s)=s+\frac{s K_{\text {on }}}{s+K_{\text {off }}} .
$$

Thus equations for the unbound concentration and flux through stratum corneum can be obtained by replacing $s$ with $g(s)$ in the above equations [30,53].

Hallmark studies of Hadgraft and collaborators [50,51] and Guy and Hadgraft [52] make them the pioneers in the use of Laplace transform for mathematical modelling of skin transport. Recently Anissimov and Roberts [53] following these ideas used the numerical inversion of Laplace transform solutions to the diffusion equation for simulations and data analysis of skin transport experiments. Bando et al. [54,55] developed a two-layer diffusion model which incorporates both polar and non-polar pathways and Laplace transformed equations were obtained to describe skin permeation of a drug or substance [30].

\subsection{Scaled particle theory}

There are three parameters that are most required to predict skin permeability, the partition coefficient, the diffusion coefficient and the path length. The partition coefficient involves partitioning between multiple phases; the stratum corneum lipid matrix and the vehicle, the keratinocytes and the vehicle, the lipids and the keratinocytes, and between the stratum corneum and deeper skin layers. The partition coefficient between the stratum corneum and the vehicle is estimated from
0.4 to 0.9 depending on the data set used in the regression.

$$
K_{\mathrm{s}, / \mathrm{v}}=a P^{b} .
$$

For estimating $K_{S C / v}$ were derived expressions that consider the individual contributions of the lipid and keratinocytes [30,56]:

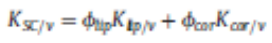

where $K_{l i p / v}$ is the lipid-vehicle partition coefficient, $K_{c o r / v}$ is the keratinocytes-vehicle partition coefficient, and $\varphi_{\text {lip }}$ and $\varphi_{c o r}$ are the volume fractions of lipid and keratinocytes in the stratum corneum, respectively, which together sum to 1 .

Scaled particle theory has been used to simplify utilization of molecular details of structures in predicting skin permeability [57]. There are two factors solute partitioning into lipid bilayers. They are the chemical and physical factors. In the chemical factor, lipid bilayer is more hydrophobic than the surrounding aqueous conditions. In the physical factor, lipid chains in the bilayer are highly organized, thus reducing the partition coefficient [30,58,59].

The theory showed that the partition coefficient of a solute from water into stratum corneum lipid matrix is comparable to that into an isotropic solvent. Thus scaled particle theory reasonably mimics the chemical environment in the stratum corneum lipid matrix. Several solvents have been used as model for stratum corneum lipids (octanol, hexadecane, olive oil, butadiene and dodecadiene). The most convenient seems to be octanol [30,56,60-65].

Mitragotri and collaborators also used this theory. They developed an equation to describe solute diffusion in lipid bilayers. The theory calculates diffusion coefficient based on the amount of work required creating a free volume to allow for solute diffusion [30].

\subsection{Homogeneous membranemodel}

The geometry of the stratum corneum and the detailed arrangement of both keratinocytes and lipids make a great deal of difference in model

calculations, as it affects the path length for diffusion, making the lipid tortuosity factor be of great importance [63,66,67]. However, since diffusion is primarily transcellular, then the lipid tortuosity factor is of the secondary importance. Also, as the keratinocytes-phase transport

reduces, the impact of lipid tortuosity increases [30].

Frasch and Barbero studied transient diffusion through model structures of the stratum corneum including both ordered and disordered keratinocyte-phases [68]. They found that diffusion was well described by a homogeneous membrane model; nevertheless, the effective diffusion coefficient, $D^{*}$, and the effective diffusion path length for the ordered structure, $h_{s c}^{*}$ : were different from the input variables [30,68]. Defining $d$ as the length, $\mathrm{t}$, as the thickness and $g$ as the space between keratinocytes, then $h_{s c}^{*}$ : is given by:

$$
h_{s c}^{*}=N(d+t+g) .
$$

Though the value of $D^{*}$ was not expressed in such manner, the flux and lag time relative are estimated by using of the microscopic flux/lag time and by effective flux/lag time [30], and their expressions of the flux and lag time relative to a homogeneous membrane, Eqs. (36) and (37) respectively, have the same microscopic lipid diffusivity $D_{0}$ and thickness $h_{0}$ :

$$
\begin{aligned}
& \frac{J_{00}}{T}=1+\frac{\propto \phi}{\sigma}+\frac{\alpha^{2} \phi^{2}}{4(1-\Phi)} \\
& \text { and } \\
& \frac{t_{\operatorname{lag} \alpha}}{t_{\operatorname{lax} 0}}=\frac{\sigma(1+\infty \Phi)}{\propto}\left(1+\frac{\propto \varphi}{\sigma}+\frac{w \quad \alpha^{2} \Phi^{2}}{1+w^{2}(1-\Phi)}\right)
\end{aligned}
$$




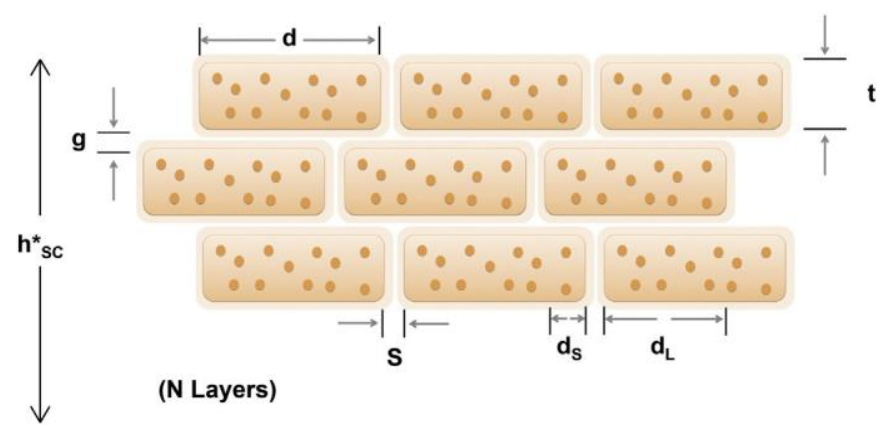

Fig. 6. Schematic diagram for the ordered structure: $h_{s o}^{*}$ effective diffusion path length for the ordered structure; $d, d_{S}$ and $d_{\mathrm{L}}$, length; t, thickness; $g$ and $s$, space between keratinocytes.

where $\propto=d / t$ is the keratinocyte aspectratio, represented by dividing the length $(d)$ by the thickness $(t)$ of the keratinocytes, and $w=d_{L} / d_{s}$ is the offset ratio. Regarding $\sigma$ and $\Phi$, they relate several variables in the ordered structure (depicted in Fig. 6): $\sigma=s / t, \quad \varphi=t /(t+g)$. Eqs. (35) and (37) were been validated for isotropic lipids and impermeable keratinocyte [68].

\section{Conclusion}

The theoretical models are very important when we are facing nanoresearch and micro research. The mathematical modelling of the skin has made notable progress in the last decades. Due to the advances in information technology the accuracy of these models steadily increases and they become easier to apply. It would be more favourable to use a single general theory applicable to any type of drug delivery system; however, there is a broad spectrum of different mathematical models, applicable to specific types of devices differing in geometry, drug and excipient type.

Fick's Law uses the concept of the passive diffusion to describe the drug delivery through the skin. The drug enters to the innermost layers of the skin through passive diffusion and this concept is the main principle used in the mathematical models.

The skin permeability is described by three parameters: partition coefficient, diffusion coefficient and path length. The first involve partitioning between multiple phases: stratum corneum and vehicle, lipids, keratinocytes, and among others. The partitioning coefficient is also called by permeability coefficient and its estimate through mathematical models can be used to predict the mass of drug that penetrates the skin from a given exposure. Several studies have reported the application of mathematical modelling for estimating permeability coefficient to calculate systemic uptake.

Two major challenges lay ahead in theoretical transdermal research. A first major challenge would be to combine the mathematical theories with models quantifying drug transport in the living organism, including drug distribution in the various organs and even within the different cell compartments. The second major challenge regards achieving a computational skin model that may validate molecular principles in dermic diffusion as a foreground of future devices.

\section{References}

[1] G. Cevc, U. Vierl, Nanotechnology and the transdermal route: a state of the art review and critical appraisal, J. Control. Release 141 (2010) 277-299.

[2] K. van der Maaden, W. Jiskoot, J. Bouwstra, Microneedle technologies for (trans)dermal drug and vaccine delivery, J. Control. Release 161 (2012) 645-655.

[3] P. Elias, Epidermal lipids, barrier function, and desquamation, J. Invest. Dermatol. 80 (1983) $44 \mathrm{~s}-49 \mathrm{~s}$.

[4] A. Arora, M.R. Prausnitz, S. Mitragotri, Micro-scale devices for transdermal drug delivery, Int. J. Pharm. 364 (2008) 227-236.
[5] J.A. Mikszta, J.B. Alarcon, Improved genetic immunization via micromechanical disruption of skin-barrier function and targeted epidermal delivery, Nat. Med. 8 (2002) 415-419.

[6] J. Gupta, E.I. Felner, M.R. Prausnitz, Minimally invasive insulin delivery in subjects with type 1 diabetes using hollow microneedles, Diabetes Technol. Ther. 11 (2009).

[7] R. Potts, V. Mak, R. Guy, M. Francouer, Strategies to enhance permeability via stratum corneum lipid pathways, Adv. Lipid Res. 24 (1991) 173-210.

[8] Y.N. Kalia, R.H. Guy, Modeling transdermal drug release, Adv. Drug Del. Rev. 48 (2001) 159-172.

[9] M.F. Wilkosz, R.H. Bogner, Transdermal drug delivery. Part 1: current status, U.S. Pharm. 28 (4) (2003).

[10] J. Kanitakis, Anatomy, histology and immunohistochemistry of normal human skin, Eur. J. Dermatol. 12 (4) (2002) 390-399.

[11] S.M. Bal, Z. Ding, E.V. Riet, W. Jiskoot, J. Boustra, Advances in transcutaneous delivery: do all ways lead to Rome? J. Control. Release 148 (3) (2010) 266-282.

[12] T. Tanner, R. Marks, Delivering drugs by the transdermal route: review and comment, Skin Res. Technol. 14 (3) (2008) 249-260.

[13] G. Chopda, Transdermal Drug Delivery Systems: A Review, available from http:// www.pharmainfo.net/reviews/transdermal-drug-delivery-systems-review2006.

[14] R.K. Subedi, Sy Oh, M.-K. Chun, H.-K. Choi, Recent advances in transdermal drug delivery, Arch. Pharm. Res. 33 (3) (2010) 339-351.

[15] Yuri G. Anissimov, Owen G. Jepps, Yuri Dancik, Michael S. Roberts, Mathematical and pharmacokinetic modelling of epidermal and dermal transport processes, Adv. Drug Deliv. Rev. 65 (2013) 169-190.

[16] Owen G. Jepps, Yuri Dancik, Yuri G. Anissimov, Michael S. Roberts, Modeling the human skin barrier-towards a better understanding of dermal absorption, Adv. Drug Deliv. Rev. 65 (2013) 152-168.

[17] M. Ai Ling Teo, C. Shearwood, K. Chye Ng, J. Lu, S. Moochhala, In vitro and in vivo characterization of MEMS microneedles, Biomed. Microdevices 7 (1) (2005) 47-52.

[18] P. Valenzuela, J.A. Simon, Nanoparticle delivery for transdermal HRT, Maturitas 73 (2012) 74-80.

[19] D. Pinaki, R.R. Patlolla, M. Singh, Interaction of nanoparticles and cell penetrating peptides with skin for transdermal drug delivery, Mol. Membr. Biol. 27 (7) (2010) 247-259.

[20] P. Connolly, C. Cotton, F. Morin, Opportunities at the skin interface for continuous patient monitoring: a reverse iontophoresis model tested on lactate and glucose, 1 (1) (2002).

[21] T.S. Ching, P. Connolly, Simultaneous transdermal extraction of glucose and lactate from human subjects by reverse iontophoresis, Int. J. Nanomedicine 3 (2) (2008) 211-223.

[22] R.L. Thangapazham, S. Sharad, R.K. Maheshwari, Skin regenerative potentials of curcumin, Biofactors 39 (2013) 141-149.

[23] M.C.Y. Heng, Wound healing in adult skin: aiming for perfect regeneration, Int. J. Dermatol. 50 (2011) 1058-1066.

[24] M. Ochalek, H. Podhaisky, H.H. Ruettinger, J. Wohlrab, R.H.H. Neubert, SC lipid model membranes designed for studying impact of ceramide species on drug diffusion and permeation-Part II: diffusion and permeation of model drugs, Eur. J. Pharm. Biopharm. 82 (2012) 360-366.

[25] M. Ochalek, H. Podhaisky, H.H. Ruettinger, R.H.H. Neubert, J. Wohlrab, SC lipid model membranes designed for studying impact of ceramide species on drug diffusion and permeation, Part III: influence of penetration enhancer on diffusion and permeation of model drugs, Int. J. Pharm. 436 (2012) 206-213.

[26] J. Siepmann, F. Siepmann, Mathematical modeling of drug delivery, Int. J. Pharm. 364 (2008) 328-343.

[27] E.B. Nauman, K. Patel, P. Karande, Design of optimized diffusion-controlled transdermal drug delivery systems, Drug Dev. Ind. Pharm. 37 (2011) 93-102.

[28] L. Simon, Repeated applications of a transdermal patch: analytical solution and optimal control of the delivery rate, Math. Biosci. 209 (2007) 593-607.

[29] L. Simon, K.S. Kim, K. Kanneganti, Effects of epidermal turnover on the dynamics of percutaneous drug absorption, Math. Biosci. 229 (2011) 16-21.

[30] S. Mitragotri, Y.G. Anissimov, A.L. Bunge, H.F. Frasch, R.H. Guy, J. Hadgraft, G.B Kasting, M.E. Lane, M.S. Roberts, Mathematical models of skin permeability: an overview, Int. J. Pharm. 418 (2011) 115-129.

[31] R. Manitz, W. Lucht, K. Strehmel, R. Weiner, R. Neubert, On mathematical modeling of dermal and transdermal drug delivery, J. Pharm. Sci. 87 (1998) 873-879.

[32] P. Schumm, C.M. Scoglio, D. van der Merwe, A network model of successive partitioning-limited solute diffusion through the stratum corneum, J. Theor. Biol. 262 (2010) 471-477.

[33] B.P. Van Milligen, On the applicability of Fick's law to diffusion in inhomogeneous systems, Eur. J. Phys. 26 (2005) 913.

[34] D. Van der Merwe, J.D. Brooks, R. Gehring, R.E. Baynes, N.A. Monteiro-Riviere, J.E. Riviere, A physiologically based pharmacokinetic model of organophosphate dermal absorption, Toxicol. Sci. 89 (2006) 188-204.

[35] D. Fitzpatrick, J. Corish, B. Hayes, Modelling skin permeability in risk assessmentthe future, Chemosphere 55 (2004) 1309-1314.

[36] T. Higuchi, Physical chemical analysis of percutaneous absorption process from creams and ointments, J. Soc. Cosmet. Chem. 11 (1960) 85-97.

[37] M.S. Roberts, S.E. Cross, M.A. Pellett, Skin Transport, in: K.A. Walters (Ed.), Dermatological and Transdermal Formulations, Marcel Dekker, New York, 2002, pp. 89-195.

[38] C. Herkenne, A. Naik, Y. Kalia, J. Hadgraft, R. Guy, Dermatopharmacokinetic prediction of topical drug bioavailability in vivo, J. Invest. Dermatol. 127 (2007) 887-894.

[39] H. Jaganathan, B. Godin, Biocompatibility assessment of Si-based nano- and micro-particles, Adv. Drug Deliv. Rev. 64 (2012) 1800-1819.

[40] N. Sekkat, Y.N. Kalia, R.H. Guy, Biophysical study of porcine ear skin in vitro and its comparison to human skin in vivo, J. Pharm. Sci. 91 (2002) 2376-2381. 
[41] Y.N. Kalia, F. Pirot, R.H. Guy, Homogeneous transport in a heterogeneous mem brane: water diffusion across stratum corneum in vivo, Biophys. J. 71 (1996) 26922700 .

[42] R.B. Walker, E.W. Smith, The role of percutaneous penetration enhancers, Adv. Drug Delivery 18 (1996) 295-301.

[43] A.C. Williams, B.W. Barry, Penetration enhancers, Adv. Drug Delivery 56 (2004) 603-618.

[44] M.L. Francoeur, G.M. Golden, R.O. Potts, Oleic acid: its effects on stratum corneum in relation to (trans)dermal drug delivery, Pharm. Res. 7 (1990) 621-627.

[45] M. Walker, J. Hadgraft, Oleic-acid-a membrane fluidizer or fluid within the membrane, Int. J. Pharm. 71 (1991) R1-R4.

[46] K. Kubota, F. Dey, S.A. Matar, E.H. Twizell, A repeated-dose model of percutaneous drug absorption, Appl. Math. Model. 26 (2002) 529

[47] R. Baker, Controlled Release of Biologically Active Agents, John Wiley \& Sons, New York, 1987.

[48] J. Crank, The Mathematics of Diffusion, Clarendon Press, Oxford, 1975.

[49] H. Carslaw, J. Jaeger, Conduction of Heat in Solids, Oxford University Press, Oxford, Great Britain, 1959

[50] J. Hadgraft, The epidermal reservoir: a theoretical approach, Int. J. Pharm. 2 (1979) 265-274.

[51] J. Hadgraft, Theoretical aspects of metabolism in the epidermis, Int. J. Pharm. 4 (1980) 229-239

[52] R.H. Guy, J. Hadgraft, A theoretical description relating skin penetration to the thickness of the applied medicament, Int. J. Pharm. 6 (1980) 321-332.

[53] Y.G. Anissimov, M.S. Roberts, Diffusion modeling of percutaneous absorption kinetics: 4. effects of a slow equilibration process within stratum corneum on absorption and desorption kinetics, J. Pharm. Sci. 98 (2009) 772-781.

[54] H.S.M. Bando, F. Yamashita, Y. Takakura, M. Hashida, In vivo evaluation of acyclovir prodrug penetration and metabolism through rat skin using a diffusion/bioconversion model, Pharm. Res. 14 (1997) 56-62.

[55] H.S.M. Bando, T. Takagi, F. Yamashita, Y. Takakura, M. Hashida, Analysis of in vitro skin penetration of acyclovir prodrugs based on a diffusion model with a metabolic process, Int. J. Pharm. 135 (1996) 91-102.
[56] J.M. Nitsche, T.-F. Wang, G.B. Kasting, A two-phase analysis of solute partitioning into the stratum corneum, J. Pharm. Sci. 95 (2006) 649-666.

[57] S. Mitragotri, A theoretical analysis of permeation of small hydrophobic solutes across the stratum corneum based on Scaled Particle Theory, J. Pharm. Sci. 91 (2002) 744-752

[58] E. Egberts, S. Marrink, H. Berendsen, Molecular dynamics simulation of a phospholipid membrane, Eur. Biophys. J. 22 (1974) 423-436.

[59] D. Tieleman, S. Marrink, H. Berendsen, A computer perspective of membranes: molecular dynamics studies of lipid bilayer systems, Biochim. Biophys. (1997) 235-270.

[60] P. Raykar, M. Fung, B. Anderson, The role of protein and lipid domains in the uptake of solutes by human stratum corneum, Pharm. Res. 5 (1998) 140-150.

[61] B. Anderson, P. Raykar, Solute structure-permeability relationships in human stratum corneum, J. Invest. Dermatol. 93 (1989) 280-286.

[62] T. Xiang, B. Anderson, The relationship between permeant size and permeability in lipid bilayer membranes, J. Membr. Biol. 140 (1994) 111-122.

[63] M.E. Johnson, D. Blankschtein, R. Langer, Evaluation of solute permeation through the stratum corneum: lateral bilayer diffusion as the primary transport mechanism, J. Pharm. Sci. 86 (1997) 1162-1172.

[64] H. Yamamoto, H. Liljestrand, Partitioning of selected estrogenic compounds between synthetic membrane vesicles and water: effects of lipid components, Environ. Sci. Technol. 38 (2004) 1139-1147.

[65] J. Kwon, H. Liljestrand, L. Katz, Partitioning of moderately hydrophobic endocrine disruptors between water and synthetic membrane vesicles, Environ. Toxicol. Chem. 25 (2006) 1984-1992.

[66] A.S. Michaels, S.K. Chandrasekaran, J.E. Shaw, Drug permeation through human skin: theory and in vitro experimental measurement, Am. Inst. Chem. Eng. J. 21 (1975) 985-996.

[67] T.-F. Wang, G.B. Kasting, J.M. Nitsche, A multiphase microscopic model for stratum corneum permeability. II. Estimation of physicochemical parameters and application to a large permeability database, J. Pharm. Sci. 96 (2007) 3024-3051.

[68] H.F. Frasch,A.M. Barbero, Steady-stateflux and lag time in the stratum corneum lipid pathway: results from finite element models, J. Pharm. Sci. 92 (2003) 2196-2207. 